\title{
Perception of distance-to-obstacle through time-delayed tactile feedback
}

\author{
Jess Hartcher-O'Brien ${ }^{1}$ Malika Auvray $^{1}$, and Vincent Hayward Fellow, IEEE ${ }^{1}$
}

\begin{abstract}
In previous vision-to-touch sensory substitution approaches, including most 'electronic white canes', typical approaches include mapping space-to-space, space-to-intensity, or space-to-frequency. To our knowledge, however, mapping space to time-delay has not been considered. Yet, because organisms must anticipate impending collisions with obstacles or anticipate being contacted by approaching objects, many organisms have developed computational short-cuts where distance-to-target is assumed to be proportional to a time-span. This short-cut often manifests itself in low-level sensorimotor behaviours and perceptual mechanisms. We studied whether untrained humans would spontaneously employ such a shortcut to estimate distance-to-obstacle in the absence of vision. The observers pressed a push button and a tactile pulse was delivered to the hand with a delay proportional to the distance to an obstacle detected by an optical range finder that they wore. The observers were not informed of the nature of the coding but could freely probe the obstacle while walking toward the target. Upon randomized presentation of obstacle distances, the observers quickly calibrated their judgement of distance-toobstacle and were able to estimate this distance within a range of four meters for a proportionality factor corresponding to a velocity of one $\mathrm{m} / \mathrm{s}$.
\end{abstract}

\section{INTRODUCTION}

The human nervous system is capable of detecting and processing an enormous variety of information sensed from the environment. The system has multiple sensors that are tuned to specific forms of environmental energy, and has developed an efficient and structured coding for these sensors (e.g.,[1]). Even though there exist modality specific optimizations, there are some general codes that have important consequences for the survival of countless species [2]. Such codes often reflect both physical constraints, as well as the evolutionary goals/imperatives of the observer. Tapping into such codes would allow one to use different modalities/sensors to detect information that is commonly just detected and processed with another sensory modality. For example, when moving through the world, information about the time-to-contact with external objects has important consequences for humans and animals alike. It affects interactions with both animate and inanimate objects. As a result, it is highly likely that a number of mechanisms have evolved to account for the time-to-contact, which is equivalent to assuming proportionality between distance and time-delay. Here we address whether humans can estimate time to contact haptically, even though it is normally accessed through vision and audition.

\footnotetext{
${ }^{1}$ Jess Hartcher-O'Brien, Malika Auvray and Vincent Hayward are with the Sorbonne Universités, UPMC Univ Paris, 06, UMR 7222, ISIR, F75005, Paris, France jesshartcher@gmail.com
}

\section{A. Universal codes}

If a code relying on mapping a distance into a time delay, or vice-versa, is universal, then it should appear in a number of species, not only humans. In fact, many species use timeto-distance or distance-to-time equivalence [3], [4], [5], [6], [7]. For example, Frost et al. have shown that specific cells in the pigeon's rotundus nucleus specifically code time-tocontact information [2]. Likewise, in heading tasks, monkeys use both odometry (vestibular cues) and visual rate of change from the visual scene to intercept a moving target [7], [5], which is also observed for humans [8]. Human observers also use a relative delay in time of arrival of the first wave front to estimate the spatial position of a sound source for auditory localisation (e.g., [6], [9]). Frogs make use of both acoustic and mechanical vibration feedback delays to estimate the distance of a competitor in the surrounding water [4]. In the auditory system, this process is low-level and totally resolvable at the level of the superior olivary complex (e.g., [10]). Thus, uncountable examples of such behaviours are evident across species, suggesting codes that rely on mapping space into time and vice-versa through moving objects or propagating waves.

\section{B. Sensory augmentation and substitution}

Visually impaired individuals have particular difficulty in ranging distant objects in space. If it is the self that needs to be located, many train themselves to use echolocation (e.g., [11]). For sighted individuals, there is a plethora of visual cues that are available to determine the distance of targets in our environment (see [12], [13]). There are monocular e.g., motion parallax, depth from motion, perspective, relative size, texture, occlusion etc. and binocular cues, e.g., stereopsis (binocular disparity), convergence, shadow stereopsis etc. [13]. A large body of research has focused on the problem of how to provide visually impaired individuals with access to the properties of the environment through an alternative sensory pathway. The devices that have been used to translate a primarily 'visual' features into information accessible to a different modality are known as sensory substitution devices (SSD). The current challenge for such devices is to define a parsimonious, plausible code that translates visual features into non-visual cues.

To introduce them in more detail, SSDs were initially designed to assist or replace specific functions of a deficient sensory modality by providing corresponding information to an alternative sensory modality. This can be achieved by converting the stimuli normally sensed through the deficient modality (e.g., light for SSDs compensating for vision) into stimuli accessible to another sensory modality (e.g., tactile 


\section{Appeared in the Proceedings of the 2015 IEEE World Haptics Conference, pp. 7-12}

vibrations or sounds). Since their inception in the sixties various kinds of devices have been developed, tested, and shown to allow their users to behave, to some degree, as if they possessed the substituted sensory organ [14], [15]. For instance, thanks to visual-to-auditory and visual-to-tactile conversion systems, blind individuals report being able to localize and recognize objects in three-dimensional space (e.g., [16], [17], [18]). However, such devices require hours and hours of training and are generally not used by their target population in daily interactions with the world.

This lack of adoption of SSDs could potentially be due to the fact that the complexity of the codes and their unintuitive nature means that learning to use the device is time consuming [19], [20]. From a nervous system perspective, the code used to translate a property of the environment into a cue in the substituting modality is arbitrary and not governed by goal directed or sensory specific constraints. Moreover, given the processing demands already made on the substituting modality, the devices can potentially undermine the ability to process non-substituted haptic/auditory cues.

\section{Challenges for providing user appropriate input}

For SSDs, and other prosthetics (e.g., [21], [22]), the current challenge is to find a code that translates the information into relevant cues/features that the nervous system can spontaneously access [3]. We suggest that distance-to-time mapping belong to this category. We implemented it into a simple device that allows users to estimate obstacle distance.

Because of the pre-existing nature of the mapping, the code should be spontaneously usable by the observer and make the target position immediately accessible. As such it should address the issue of long training times required to learn the mapping between object feature and sensorially substituted information. The second challenge of leaving the substituting modality in tact for non-substituted inputs is also met by the form of feedback we provide (see II-B). If users are spontaneously able to make use of the mapping that the device provides between object distance and feedback delay, then this might open up a whole new direction in sensory substitution device development.

\section{PROCEDURE}

\section{A. Observers}

Eight sighted observers took part in the current experiment. All of them volunteered their time and none had previously participated in related studies. The observers were also naive to the purpose of the study. The group consisted of five male and three female observers, with mean age ( \pm standard deviation) of $28.7( \pm 4.6)$ years. According to the Cohen's handedness test, all were right handed.

\section{B. Apparatus and stimuli}

We developed a device that provides haptic feedback about object distance via a short pulse vibration. The device, shown in Fig. 1, consisted of a laser rangefinder (SF02/F, LightWare, South Africa) secured to a bicycle helmet coupled to a wide-band vibrotactile transducer (Haptuator, Tactile Labs,
Canada) with millisecond-level temporal resolution attached to the handle. A membrane switch button which was used by the observer to initiate probing of the environment was on the upper surface of the handle. A button press triggered a reading from the sensor which was then translated it into a short, $10 \mathrm{~ms}$ tactile click via the transducer. The onset delay was proportional to the distance estimate from the rangefinder. The rangefinder provided precise distance estimates within a $0-40 \mathrm{~m}$ range with an accuracy of $\pm 0.1 \mathrm{~m}$. The proportionality factor between the sensed distance and the feedback delay was set to $1.0 \mathrm{~m} / \mathrm{sec}$ for distances between $0.5 \mathrm{~m}$ and $4.0 \mathrm{~m}$. We verified of the device to test that the function defining the mapping between target distance and feedback delay after button press were as we predicted. The device responded with a delay of $\Delta t=1 / v \Delta l$ where $\Delta l$ was the sensed distance and $1 / v$ a proportionality factor. We verified the reliability of the device with 5 independent clocking measurements made from $0.5 \mathrm{~m}$ to $3.5 \mathrm{~m}$ by steps of $0.5 \mathrm{~m}$. The linearity between distance and delay had a coefficient of determination $r=0.96$ and the standard deviation was $\sigma=0.02 \mathrm{~m}$.

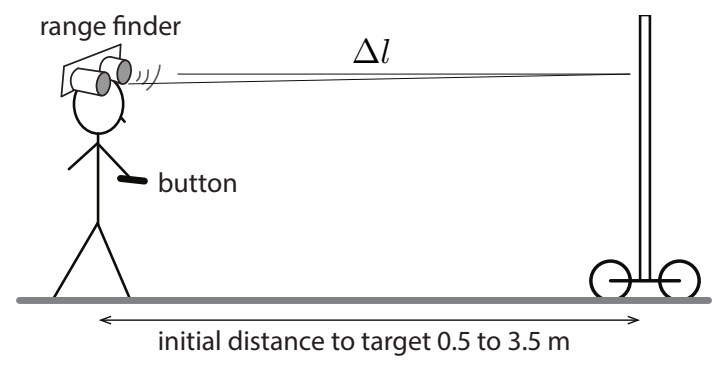

Fig. 1: Schematic representation of the setup and device. The target could initially be between 0.5 to 3.5 meters from the observer. When the observer pressed the button, the laser rangefinder estimated the target distance, $\Delta l$, and within $80 \mathrm{~ms}$, the device converted the distance into a haptic feedback click delayed by $1.0 \mathrm{~m} / \mathrm{s} \times \Delta l$ delivered to the observer's hand.

Observers took the handle in their right hand. The vibrotactile intensity was constant, and on average $2.5 / \mathrm{g}$ and was $10 \mathrm{~ms}$ in duration. The target stimulus, sensed by the device, was a moveable flat board, $1.5 \times 2.5 \mathrm{~m}$ (see Fig. 1 ). The matt surface of the board was easily detectable by the laser rangefinder. The distances were marked out on the floor relative to the observer starting point at $0 \mathrm{~m}$ and the board was silently displaced on a trial-by-trial basis. During the experiment, white noise was played through ear-buds to the observer to mask any task related auditory cues.

\section{Main Task}

Throughout the experiment, observers were blindfolded and wore the range-finder helmet that was attached to the processing unit inside the handle that they held in their dominant hand. The relationship between object distance and the haptic feedback provided to the observer was never made explicit. The only information provided in the experimenter 
instructions was that when observers probed the environment in search of the target, haptic feedback, in the form of a short pulse would be given. Observers were to use this feedback to estimate target distance. In a short calibration phase, observers experienced the bounds of the distances used during the experiment: $(0.5 \mathrm{~m}$ and $3.5 \mathrm{~m})$. Each bound was probed three times with the device, providing the distanceto-delay space. The experiment consisted of three phases and lasted approximately one hour.

a) Pre-test: At the beginning of the task, the observer stood upright, chin tucked in slightly towards the chest, such that the rangefinder, mounted on the helmet which was securely fastened to the observer's head, was pointing straight ahead to the target. The observer gripped the handheld component of the device, with the thumb resting on the membrane switch button. Prior to the commencement of each trial, the experimenter manually moved the target to a pre-defined, randomized distance from the observer startpoint. The experimenter tapped the observer's right shoulder to signal that the trial could begin.

On the signal, the observer pressed the button to probe for the target. A single pulse was given as feedback and, using the feedback, the observer was instructed to walk until one meter from the target. While walking, the observer could probe at self-determined intervals, to update their estimate of the relative position of the target. When the observer estimated that they were at one meter from the target they stopped and signalled it by pressing the button on the handle a final time. The actual distance was taken from the final probe estimate from the device. The observer was then guided back to another initial start position. Once the target position had been updated for the following trial, a new trial would begin. There were forty-nine trials in this task, constituting seven repetitions of each of the seven target distance tested.

b) Free navigation: In the second phase of the experiment, the observers navigated freely along a $25 \mathrm{~m}$ narrow corridor and used self-generated feedback, identical to that used during the test phase, by pressing the button to probe the environment. The observer's goal was to reach the end of the corridor with minimum contact with external surfaces. In this case, the observer was trying to optimize long feedback delays, until almost at the end-point of the corridor.

c) Post-test: The post-test was identical to the pre-test in that the observer's task was to "walk up to one meter from the target", as it was in the pre-test phase. The same seven target distances were used with the same number of repetitions.

\section{Control task}

Two of the eight observers ran a control task to provide an indication of the baseline noise in their odometric, vestibular distance estimation accuracy and distance judgement when walking up to one meter from the target. In this task, the observer again wore the helmet device, the blind-fold and ear buds. At the commencement of each trial, the observer stood at the initial start point and visually assessed the distance of the target. The target could be at any of the seven distances and was repeated seven times. After viewing the distance of the target the observer adjusted the blindfold and walked until they estimated they were one meter from the target.

\section{E. Data analysis}

The raw data was converted into a distance estimate by subtracting one meter from the final position of the observer relative to the target at the end of the trial. All the data are therefore reported in the distance walked by the subject.

\section{RESULTS}

Our goal was to investigate the extent to which observers were able to immediately understand and use the haptic feedback delay to estimate the position of an object in depth. If the feedback is intuitive, then results should be better than chance in the absence of explicit instructions. In addition, with an optimal feedback code there should be no difference between the target distance and the error in the walked distance. Furthermore, if indeed a pre-existing code was used by the observers, we would predict that there should be no difference between the distance estimates in the pre- and post-test phases.

To investigate whether our data show that the distance-todelay mapping was spontaneously available to the observers, we tested whether the variance in distance estimates differed significantly from the target distance of one meter with a single-sample t-test for the pre-test phase $t(55)=0.39$, $p>0.05$, collapsing across initial distance. We did the same thing for the post-test results $t(55)=1.49, p>0.05$. In both cases the distance estimates did not differ significantly from the target distance of one meter, suggesting that the cue was easily accessible to the observers and that they were able to use it to accurately walk until one meter from the target. Using the current mapping between feedback delay and target distance, observers need less than 50 trials to effectively navigate their environment, walk the appropriate distance, and avoid collision with the target object. This is in stark contrast to training times required for proficient use in other studies [17], [19].

In addition, we wanted to see whether the pre-existing nature of the code between object distance and feedback delay would make training and longer exposure unnecessary. The pre- and post-test results show no significant difference $t(1,101)=-0.6638, p>0.05$ which confirms that training does not provide a significant improvement given the already low error rate in the pre-test.

Figure 2 shows the mean distance estimation errors at each initial distance for the pre- and post-tests. The error bars show the variance across observers for each initial distance. It is evident that there was no significant difference in the variance between the two conditions when we collapsed across the initial target distance, $\chi=1.58, p=0.2$. When we looked at the individual observer estimates as a function of each distance, again there was no significant difference, although there was some variation at the specific distances for individual observers, $F(1,7)<1, p>0.05$. As seen in 
the box plots in Fig. 2 the variance in distance estimation decreased for most observers in the post-test as demonstrated by the overall decrease in range of the estimates, however, this difference was not significantly different across the two test phases. Therefore, we see evidence that the pre-existing nature of the code allows observers to spontaneously access the distance of an object from the simple haptic feedback. No significant training or exposure is required beyond the initial calibration to the distance-delay space.

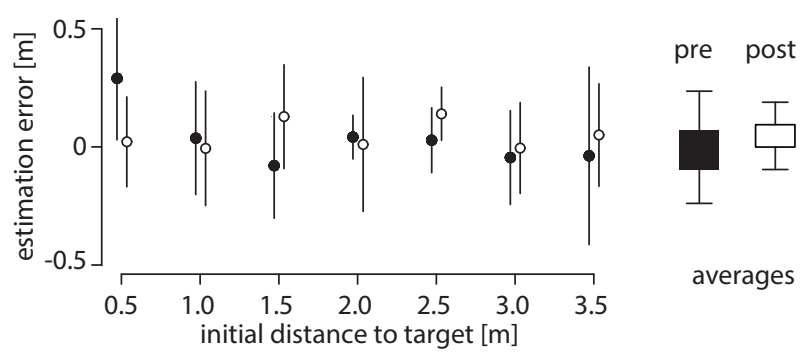

Fig. 2: Mean distance estimation error in the walk-onemeter-from-target task, for each initial distance tested in the pre-test (black filled circles), the post-test (hollow circles), averaged across observers. The error bars represent the 95\% confidence intervals. Estimation error for the pre-test (black), and the the post-test (white), averaged across initial distances. The error bars represent interquartile ranges.

We verified the nature of the relationship between pre- and post-test results, see Fig. 3. A regression line fitted to the data has a slope of $0.44(95 \%$ C.I. $=0.2-0.7)$ and an intercept of 0.12 (C.I. $=0.02-0.13$ ) with $R^{2}=0.0652, p=0.0575$ indicating that the pre-test results were not a significantly powerful predictor of post-test error. From Fig. 3 it is evident that there was a large spread in the relationship between the two test phases for the different distances, suggesting that any variability in the data was due to random variability of observer performance in the task.

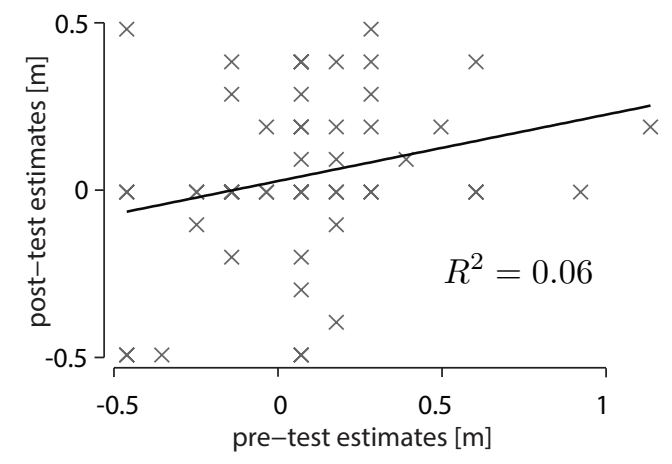

Fig. 3: Mean $\delta$ distance estimates in the walk-one-meterfrom-target task, for each initial distance tested in the pretest and post-test, for each observer, with the regression line of best fit given (black line).

The variance in the estimated one meter from the target data was very small both for pre- and post-test conditions. In order to investigate whether the source of the variance could be attributed to error in odometry, we ran two control subjects to see how they performed when visually assessing the position and then, blindfolded, walked up to one from the target. Figure 3 shows the distance estimation errors for all three conditions for these two subjects as a function of initial target distance.
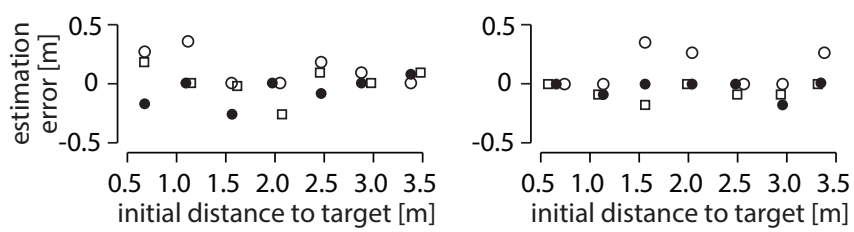

Fig. 4: Mean distance estimates in the walk-one-meter-fromtarget task, for each initial distance tested in the pre-test (filled circles), the post-test (hollow circles) and the control task (squares), for two observers who completed the control task where distance was assessed visually.

The control sample size was too small to statistically verify the similarity across tasks, but from Fig. 4 it is clear that the results from the three tests, pre- post- and control, all covary. Therefore, the variability in the data obtained by using the haptic feedback is unlikely to differ significantly (for a larger sample) from walking after visually assessing target position.

\section{Discussion}

\section{A. Overall Results}

The question addressed by this study is whether or not observers can use tactile information to judge the distance of a target (in the absence of contact with the target), if the skin receives a short vibrational pulse with a delay proportional to the distance of the sensed target. From the task walk-untilone-meter-from-target, observers were able to self-calibrate their perception of target position based on odometry and delay. The continuous probing provides something analogous to an haptically sensed looming sensation. Observers clearly had spontaneous access to the mapping between target distance and feedback delay given the lack of error in the pretest data. We would argue that this is possible because of the pre-existing nature of the code we used to translate a spatial property into a readily accessible form of information for the brain. It is clear that the observers could solve for target distance given that the relationship between target distance and the feedback delay is linear.

The time delay cue we provided via the device is dynamic in that it depends on the distance-delay mapping space sampled in the calibration phase, as well as the history of delays experienced in previous trials during the task. In the current task, there was very little variance in the estimated one meter, making it unnecessary to look into the history of trials as they influence the current estimate. However, in future studies we will look at absolute distance estimates and higher-level tasks such as shape discrimination where the influence of dynamic updating may exert an even stronger influence. The present results are strongly supportive of the usability of the 
device. As an additional verification of the validity of the cue provided by the device, for two control subjects, performance was also comparable to the task using visual input in place of haptic feedback for distance estimation.

\section{B. Improvements over previous SSD implementations}

The advantages of our approach for navigation, compared to general SSD systems are readily apparent: no training is needed and users navigate at a standard comparable to vision, as is evident in Fig. 4.

A large number of previous visual-to-tactile and visualto-auditory cue SSDs have used artificial codes to provide the user with abilities such as object recognition, without being concerned specifically with coding object distance. This is the case for visual-to-tactile conversion systems such as the "Tongue Display Unit" which uses an analogical code to convert visual images into stimulation applied to the user's tongue. With this system users are able to explore their environment, approximate an object's two dimensional position and it's shape within an hour or two of training, extending to eight hours for better than chance performance [23].

Visual-to-auditory conversion systems such as 'the vOICe', the "Prosthesis for Substitution of Vision by Audition", and the Vibe [24] convert three dimensions of a visual image (vertical position, horizontal position and brightness) into three dimensions of the auditory signal (usually frequency, inter-aural disparity, time scanning or loudness, see [14]). These devices require long training times, on average fifteen hours before the user can integrate the substituted information for improved task performance. In this class of conversion systems, depth is not coded directly and remains difficult for the user to access.

A number of devices have also focused on coding spatial position, i.e. direction and distance of a target. However, the codes used are more artificial than the one implemented in the current device. Through an infra-red light emission/reception system the Tom Pouce technology allows its users to receive tactile feedback about the presence of an obstacle with about $20 \%$ error in the estimated distance it provides. This is a high margin of error to deal with at short distances.

Further device examples provide auditory feedback and code distance via pitch, and the horizontal position of the target by inter-aural disparity (e.g., UltraSonic Torch, Sonic Glasses [25], [26]; Sonic Pathfinder [27]). The Télétact uses a laser to detect obstacles, with two modes of feedback, acoustic and tactile. The tactile feedback condition presents increasing/decreasing intensity of pulses dependent on distance of the obstacle detected by the laser rangefinder. Decreasing intensity specifies targets at a longer distance. In the acoustic feedback mode, the distance of the target is mapped to increasing/decreasing pitch of tones. Closer obstacles produce higher pitch feedback. This system is able to provide the distance to an obstacle with $1 \%$ error in an area ranging from $10 \mathrm{~cm}$ to $10 \mathrm{~m}$ from the user. However, it's main limitation is the difficulty of use and the time required to establish the mapping between distance and pitch height/intensity. To be able to use the feedback for navigation requires a manual scan of the environment that involves the user learning how their proprioceptive state is linked to object position and acoustic/tactile feedback. The code implemented is not intuitive and requires extensive training [20].

Finally, the EyeCane translates point-distance information into tactile cues. It provides the user with distance information for detecting nearby ground level obstacles $(0-1 \mathrm{~m})$ and its basic functioning can be acquired after a short 23 min training period, however, it has only been employed in virtual environments. It remains to be seen whether the cue use can be generalised to real environments and to nonground level objects. Neither of the latter two devices use an existing biological mapping in the same way as implemented for the current device.

\section{Biological Vs. Artificially Imposed Codes}

The main advantage of our mapping distance to time delay is that it does not impose a novel transformation between features across sensory channels. It uses a preexisting biological code manifested by numerous time-space permutations [3]. The pre-existing and somewhat universal nature of the code means that it should not matter if the feedback is haptic, as in our study, or auditory. In both cases we would predict the same result.

In relation to the universal nature of this mapping, how it is used by an organism depends on its evolutionary goal. That is, when a human observer is navigating, then the perceived distance to a target or obstacle is highly relevant information. For visual information processing this appears obvious. For many species, including humans, the image of an object looming toward them typically elicits an escape or avoidance response. This has been demonstrated experimentally in fiddler crabs, fishes, frogs, turtles, chicks, monkeys and humans [3]. Moreover, these studies demonstrate the lowlevel automatic nature of the responses, in that they remain essentially invariant even when the looming object's size, shape and starting distance are changed. Several other studies have shown, in a variety of species, that locomotor behaviour of the moving animal itself toward stationary features in its environment is also controlled by monocular information associated with the rapid expansion of features in the retinal image. Here we show that haptic feedback about the relative distance of an object can be used in a similar fashion, providing automatic low-level responses. As the device was mounted on the head, any angle changes in probing were coded in the efferent copy of the observers head movements during walking and therefore able to be accounted for in the final distance estimate.

\section{ACKNOWLEDGEMENT}

This work was supported by a European Research Council (FP7 program) ERC Advanced Grant PATCH (no. 247300) to V.H. and a Fyssen Foundation post-doctoral fellowship awarded to J.H.O. M.A. is funded by Agence Nationale de la Recherche (ANR-11-JSH2-003-1). 


\section{Appeared in the Proceedings of the 2015 IEEE World Haptics Conference, pp. 7-12}

\section{REFERENCES}

[1] D. Goldreich, "A Bayesian Perceptual Model Replicates the Cutaneous Rabbit and Other Tactile Spatiotemporal Illusions," PLoS ONE, vol. 2, no. 3, p. e333, Mar. 2007.

[2] H. Sun and B. J. Frost, "Computation of different optical variables of looming objects in pigeon nucleus rotundus neurons," Nat Neurosci, vol. 1, no. 4, pp. 296-303, 1998.

[3] B. J. Frost and H. Sun, "Chapter 2 The biological bases of timeto-collision computation," in Advances in Psychology, ser. Time-toContact, H. H. Savelsburgh and G. J. P., Eds. North-Holland, 2004, vol. 135, pp. 13-37.

[4] W. Halfwerk, R. A. Page, R. C. Taylor, P. S. Wilson, and M. J. Ryan, "Crossmodal Comparisons of Signal Components Allow for RelativeDistance Assessment," Curr. Biol., vol. 24, no. 15, pp. 1751-1755, 2014.

[5] A. Chen, G. C. DeAngelis, and D. E. Angelaki, "Functional Specializations of the Ventral Intraparietal Area for Multisensory Heading Discrimination," J. Neurosci., vol. 33, no. 8, pp. 3567-3581, 2013.

[6] J. Blauert, Spatial Hearing: The Psychophysics of Human Sound Localization. MIT Press, 1997.

[7] G. C. DeAngelis and D. E. Angelaki, "Visual-Vestibular Integration for Self-Motion Perception," 2012.

[8] J. Speigle and J. Loomis, "Auditory distance perception by translating observers," in Virtual Reality, 1993. Proceedings., IEEE 1993 Symposium on Research Frontiers in, 1993, pp. 92-99.

[9] F. L. Wightman and D. J. Kistler, "Resolution of front-back ambiguity in spatial hearing by listener and source movement," J. Acoust. Soc. Am., vol. 105, no. 5, pp. 2841-2853, 1999.

[10] G. Harnischfeger, G. Neuweiler, and P. Schlegel, "Interaural time and intensity coding in superior olivary complex and inferior colliculus of the echolocating bat Molossus ater," J. Neurophysiol., vol. 53, no. 1, pp. 89-109, 1985.

[11] T. Papadopoulos, D. S. Edwards, D. Rowan, and R. Allen, "Identification of auditory cues utilized in human echolocation-Objective measurement results," Biomedical Signal Processing and Control, vol. 6, no. 3, pp. 280-290, 2011.

[12] J. M. Loomis and D. W. Eby, "Perceiving Structure From Motion: Failure Of Shape Constancy." in ICCV, 1988, pp. 383-391.

[13] S. E. Palmer, Vision science: Photons to phenomenology. Cambridge, MA, US: The MIT Press, 1999, vol. xxii.

[14] M. Auvray and E. Myin, "Perception With Compensatory Devices: From Sensory Substitution to Sensorimotor Extension," Cogn. Sci., vol. 33, no. 6, pp. 1036-1058, 2009.

[15] A. Bubic, E. Striem-Amit, and A. Amedi, "Large-Scale Brain Plasticity Following Blindness and the Use of Sensory Substitution Devices," in Multisensory Object Perception in the Primate Brain, J. Kaiser and M. J. Naumer, Eds. Springer New York, 2010, pp. 351-380.

[16] M. Auvray, S. Hanneton, and J. K. O’Regan, "Learning to perceive with a visuo - auditory substitution system: Localisation and object recognition with 'The vOICe'," Perception, vol. 36, no. 3, pp. 416430, 2007.

[17] P. Bach-Y-Rita, C. C. Collins, F. A. Saunders, B. White, and L. Scadden, "Vision Substitution by Tactile Image Projection," Nature, vol. 221, no. 5184, pp. 963-964, 1969.
[18] S. Levy-Tzedek, S. Hanassy, S. Abboud, S. Maidenbaum, and A. Amedi, "Fast, accurate reaching movements with a visualto-auditory sensory substitution device," Restor. Neurol. Neurosci., vol. 30, no. 4, pp. 313-323, 2012.

[19] N. A. Giudice, R. L. Klatzky, C. R. Bennett, and J. M. Loomis, "Perception of 3-D location based on vision, touch, and extended touch," Exp Brain Res, vol. 224, no. 1, pp. 141-153, 2013.

[20] J. M. Loomis, R. G. Golledge, and R. L. Klatzky, "Navigation System for the Blind: Auditory Display Modes and Guidance," Presence: Teleoperators and Virtual Environments, vol. 7, no. 2, pp. 193-203, 1998.

[21] J. K. Chapin, "Using multi-neuron population recordings for neural prosthetics," Nat Neurosci, vol. 7, no. 5, pp. 452-455, 2004.

[22] G. A. Tabot, J. F. Dammann, J. A. Berg, F. V. Tenore, J. L. Boback, R. J. Vogelstein, and S. J. Bensmaia, "Restoring the sense of touch with a prosthetic hand through a brain interface," PNAS, vol. 110, no. 45, pp. 18 279-18284, 2013.

[23] K. Kaczmarek and S. Haase, "Pattern identification and perceived stimulus quality as a function of stimulation waveform on a fingertipscanned electrotactile display," IEEE Trans. Neural Syst. Rehabil. Eng., vol. 11, no. 1, pp. 9-16, Mar. 2003.

[24] S. Hanneton, M. Auvray, and B. Durette, "The Vibe: a versatile visionto-audition sensory substitution device," Appl. Bionics Biomech., vol. 7, no. 4, pp. 269-276, Dec. 2010.

[25] L. Kay, "An ultrasonic sensing probe as a mobility aid for the blind," Ultrasonics, vol. 2, no. 2, pp. 53-59, Apr. 1964.

[26] _ _ "Sensory AIDS to Spatial Perception for Blind Persons: Their Design and Evaluation," in Electronic Spatial Sensing for the Blind, ser. NATO ASI Series, P. D. H. Warren and D. E. R. Strelow, Eds. Springer Netherlands, 1985, no. 99, pp. 125-139.

[27] A. G. Dodds and A. Others, "The Sonic Pathfinder: An Evaluation," J. Vis. Impair. Blind., vol. 78, no. 5, pp. 203-6, Jan. 1984. 